\title{
COMPARISON OF THE EFFECTS OF CO- TRANSPLANTATION OF BONE MARROW HEMATOPOIETIC STEM CELLS AND THYMIC MULTIPOTENT STROMAL CELLS ON THE IMMUNE SYSTEM OF MICE DEPENDING ON METHODS
}

\author{
Demchenko Dariia ${ }^{1} \bowtie$ \\ chekhdariia@gmail.com \\ Nikolskiy Igor ${ }^{1}$ \\ Nikolskaya Valentyna ${ }^{1}$ \\ Pelykh Natalia ${ }^{2}$ \\ ${ }^{1}$ Department of Cell and Tissue Technologies \\ State Institute of Genetic and Regenerative Medicine \\ of the National Academy of Medical Sciences of Ukraine \\ 67 Vyshgorodskaya str., Kyiv, Ukraine, 04114 \\ ${ }^{2}$ Department of Veterinary Medicine, Hygiene and Animal Breeding \\ Kherson State Agrarian and Economic University \\ 23 Stritenska str., Kherson, Ukraine, 73006
}

$\triangle$ Corresponding author

\section{Abstract}

Physical interaction of multipotent stromal cells (MSCs) and hematopoietic stem cells (HSCs) is a modern approach to effective and focused changes in the properties of HSCs. Resulting of those contact interaction is significant activation of cells with following immune system restoration.

The purpose of the study is to investigate the effect of co-transplantation of bone marrow hematopoietic stem cells (HSCs) and thymic multipotent stromal cells (MSCs) separately and as a union of cells on regeneration of the murine immune system, damaged by cyclophosphamide.

MSCs were obtained from thymuses of C57BL mice using explant technique. Bone marrow cells (BMCs) were obtained by flushing out the femur with a nutrient medium. BMCs were cocultivated for 2 hours on the monolayer of thymus-derived MSCs. The immune deficiency of mice was modelled by the treatment with cyclophosphamide (CP). After that, the cells were co-transplanted in two methods (separately into different the retroorbital sinus and as a union after co-cultivation) and the parameters of the immune system were evaluated. It was shown, that separate co-transplantation of BMCs and thymus-derived MSCs is associated with the restoration of the number of bone marrow cells, thymus, spleen and lymph nodes with an increase in the proliferation index of lymph node cells by 1.4 times compared to control. It normalized the previous reduced concentration of hemoglobin and hematocrit in the blood. Co-transplantation had a suppressive effect on the blast transformation reaction, induced by phytohemagglutinin, by 4.3 times, but showed a stimulating effect on DTHR response by 1.6 times compared to control.

Co-transplantation of the union of BMCs and MSCs is associated with the restoration of the number of bone marrow cells, spleen and lymph nodes. The level of spontaneous apoptosis of lymph node cells significantly increased by 3.3 times compared to control. It had not effect on hematological parameters, but is activated to impact the immune system. Thus, as a result of cells union administration showed normalization of the bactericidal activity of peritoneal macrophages, 
unlike the separate co-transplantation. This cells graft had a suppressive effect on the number of antibody-producing cells in the spleen by 4.2 times compared to control.

Previous co-cultivation and contact interaction of cells change the properties of cell graft. The effect of co-transplantation of BMCs and thymic MSCs is not a simple additive effect of cells. It is acquiring the features typical to certain cell types, and the expression of new characteristics. We assume this phenomenon as a result development of complex cells cooperative processes in vivo and in vitro.

Keywords: bone marrow cells; thymic multipotent stromal cells; co-transplantation; regeneration; immune system.

DOI: $10.21303 / 2504-5695.2021 .001993$

\section{Introduction}

Regeneration of the immune system, caused by different insults, remains a current issue of modern biology and medicine. Researchers often focus on the study of hematopoietic stem cells (HSCs), their progenitor cells and multipotent stromal cells (MSCs) of various organ origin [1, 2]. HSCs are the cells that produce all mature hematopoietic cells and MSCs provide a necessary microenvironment in the thymus and bone marrow (BM) and probably in the peripheral lymphoid organs for normal functioning of HSCs [3]. Meanwhile, most of the research papers are devoted to BM-derived MSCs, which shaping the most important for the functioning of hematopoietic cells structures called "niches" [4]. There are much fewer data on thymus-derived MSCs.

It is postulated, that there exist the thymic niches, in which, with an involvement of MSCs, the conditions are created for differentiation of the T-cell progenitors, migrating to the thymus from the bone marrow [5]. Thymic mesenchyme is necessary for its embryonic morphogenesis and plays a direct role in the lymphopoiesis and cell migration in the thymus [6]. Thus, it is obvious, that the interaction between MSCs and HSCs plays a key role in the tissues of the central organs of the immune system. However, the MSCs are also important in the course of their interaction with the hematopoietic cells on the periphery. They suppress the post-transplant reactions [7] and stimulate antibodies synthesis, and also restrain the processes, associated with the proliferation of T-lymphocytes in the different in vitro systems. Thus, the MSCs affect not only HSCs but also the various populations and subpopulations of the lymphocytes.

The MSCs of various origin are known to be rather heterogeneous and to differ in terms of their activeness, most apparently on account of their links with the microenvironment [1,8]. As a result, the set of properties of the MSCs of the thymus, the central organ of immunity, is probably of significant importance for the recovery of the immune system. One of the new approaches to effective and focused changes in the properties of HSCs can be the use of physical interaction of thymic MSCs and HSCs. It is leading to significant activation of interacting cells. This activation could be the basis for developing more effective methods to restore the immune system in the future.

Thus, the objective of the research paper was to investigate the effect of co-transplantation of bone marrow HSCs and thymic MSCs separately and as a union on regeneration of the murine immune system, damaged by cyclophosphamide.

\section{Materials and methods}

Experiments were conducted on male C57BL mice aged 6-8 weeks and weighing 18-20 g from the vivarium of Kavetsky Institute of Pathology, Oncology and Radiobiology of the National Academy of Sciences of Ukraine, which received a balanced diet and had free access to water ad libitum. All studies on experimental animals were carried out in compliance with the requirements of Article 26 of the Law of Ukraine «On the Protection of Animals from Cruelty» (February 21, 2006) and the European Convention for the Protection of Vertebrate Animals Used for Experimental and Other Scientific Purposes (Strasbourg, 1986).

After euthanasia of animals by cervical dislocation under ether anesthesia, thymuses and femurs, spleens and mesenteric lymph nodes were isolated under sterile conditions as well as peripheral blood was collected. 
BMCs were obtained by flushing of the femoral diaphysis with a nutrient medium. Thymus-derived MSCs were obtained from C57BL/6 male mice at the age of 6-8 weeks by the standard explants technique [9]. Cultivation was carried out in DMEM/F12 (Sigma, USA), supplemented with $10 \%$ fetal bovine serum FBS (Sigma, USA), $10 \mathrm{mM}$ L-glutamine (Sigma, USA) and $100 \mathrm{IU} / \mathrm{mL}$ penicillin and $100 \mu \mathrm{g} / \mathrm{mL}$ streptomycin (Darnytsya, Ukraine) in a $\mathrm{CO}_{2}$ incubator (Jouan, France) at $37{ }^{\circ} \mathrm{C}$ and $5 \%$ in the $\mathrm{CO}_{2}$ atmosphere. Subcultivation of cells in the ratio 1:3 was carried out using a mixture of $0.05 \%$ trypsin (Biotestmed, Ukraine) and $0.02 \%$ EDTA (Sigma, USA) solutions. The obtained cells were attached to plastic, had fibroblast-like morphology and formed colonies. The characteristics of MSCs were additionally established by inducing their differentiation into the osteogenic and adipogenic directions in the specific media [10-13].

To study the effect of cell transplants on regeneration of the murine immune system, four groups were formed:

- I group - control, normal animals $(n=10)$, treated with water for injection intraperitoneally and after 2 hours $0.1 \mathrm{~mL}$ of the nutrient medium DMEM/F12, 1:1, (Sigma, USA) supplemented with $5 \%$ syngeneic murine serum;

- II group - mice, treated with $200 \mathrm{mg} / \mathrm{kg} \mathrm{CP}$ intraperitoneally $(n=9)$;

- III group - animals, treated with CP with following co-transplantation of $10^{6} \mathrm{BMCs}$ with $5 \times 10^{4} \mathrm{MSCs}$ in $0.1 \mathrm{~mL}$ of the nutrient medium separately into different retroorbital sinus $(n=7)$;

- IV group - animals, treated with CP with the following co-transplantation of the union of $10^{6}$ BMCs with $(5-18) \times 10^{4}$ MSCs $(n=6)$.

Cells were injected 2 hours after the CP treatment. From this type of co-transplantation BMCs were obtained by incubation of BMCs on thymus-derived MSCs monolayer for 2 hours. Then BMCs, detached from thymus-derived MSCs, were collected by light pipetting. After resuspension, BMCs with attached to thymus-derived MSCs were collected using Versen's solution (the fraction was designated as the union of BMCs and MSCs).

In a week, mice of all groups were immunized intraperitoneally with $1 \times 10^{8}$ sheep erythrocytes. After 4 days, a repeated injection of the same number of cells subcutaneously into the footpad was performed. After a day, the number of antibody-producing cells was measured by local hemolysis in gel. The delayed hypersensitivity rate was measured by the difference in the mass of the experimental and control feets. The control mice were immunized according to the same scheme. Thus, the study of the immune system of animals in all groups was carried out 12 days after the cyclophosphamide treatment, considering the dynamics of its immunosuppressive effect.

The hematocrit, hemoglobin concentration in the peripheral blood was determined by the automatic hematologic analyzer Particle Counter PCE-210 (ERMA Inc., Japan). The analysis of the cell cycle and apoptosis of cells from mesenteric lymph nodes was performed by propidium iodide staining using FACScan flow cytometer (Becton Dickinson, USA), counting $25 \times 10^{3}$ cells per sample. ModFit LT software was used to establish the distribution of cells in the cell cycle phases [14].

The study of proliferative and cytotoxic activity of natural killer lymphocytes was performed by the Mossman method [15]. The phagocytosis activity of peritoneal macrophages with FITC-labeled $S$. aureus was investigated by flow cytometry [16]. The level of spontaneous and induced bactericidal activity of peritoneal macrophages was determined by their ability to recover nitro blue tetrazolium (NBT) [17].

The obtained results are processed by the methods of variation statistics using MS Office Excel (Microsoft, USA) software. For quantitative parameters, mean and standard error of mean $(M \pm m)$ were calculated. The non-parametric Mann-Whitney U test was used to determine the significance of the differences between the compared groups. The critical value of the significance level was considered $p<0.05$.

\section{Results and discussion}

Twelve days after the CP treatment, the body weight of mice was reduced by $13 \%(16.8 \pm 0.8 \mathrm{~g}$ vs. $19.3 \pm 0.4 \mathrm{~g} ; p<0.05)$ compared to the control group of intact animals. This may be the result of the common toxic effect of $\mathrm{CP}$. The administration of $\mathrm{CP}$ resulted in a $36 \%$ reduction in bone marrow cellularity $(p<0.05)$ compared to the control group of intact animals that did not recover on the 
$12^{\text {th }}$ day. Parameters of mice after both types of co-transplantation in this term did not differ from the norm, indicating their positive effect on the recovery of BM by the $12^{\text {th }}$ day $($ Fig. $\mathbf{1}, \boldsymbol{a})$.

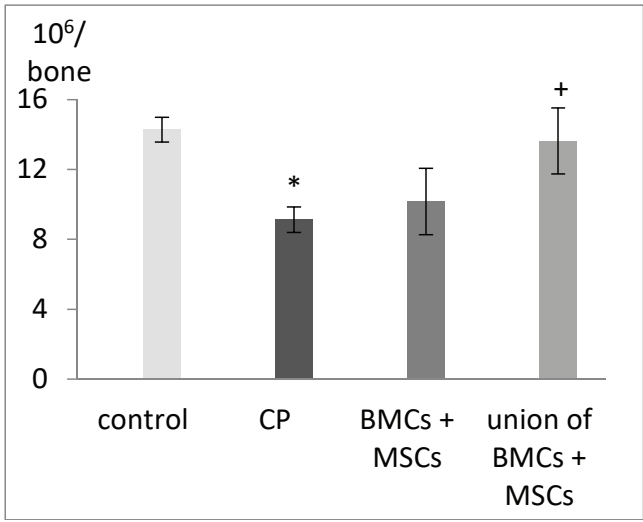

$a$

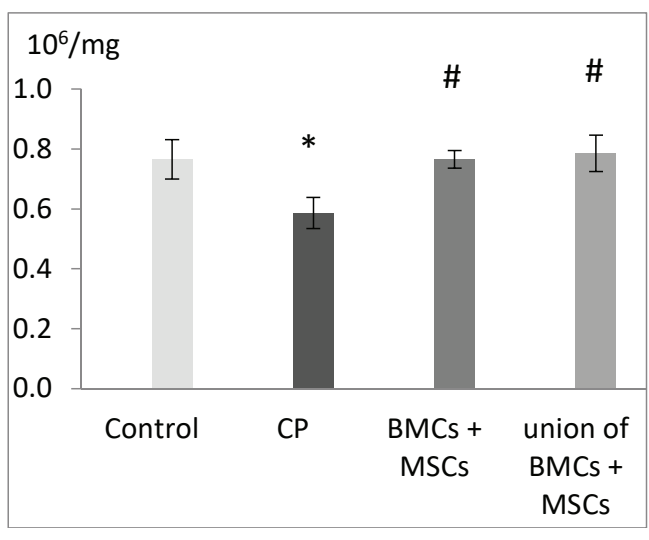

$c$

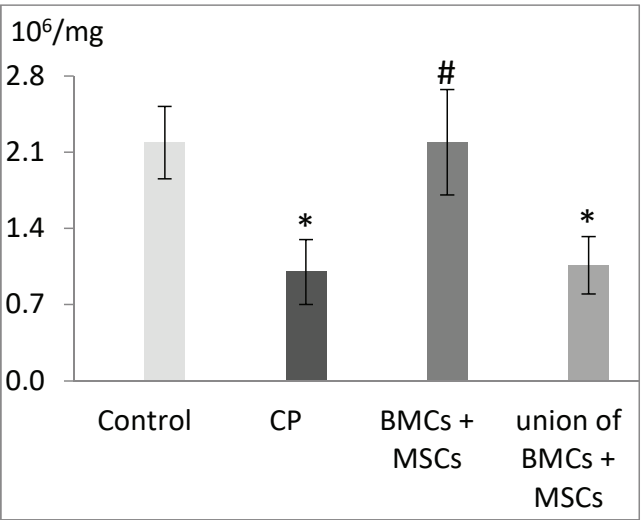

$b$

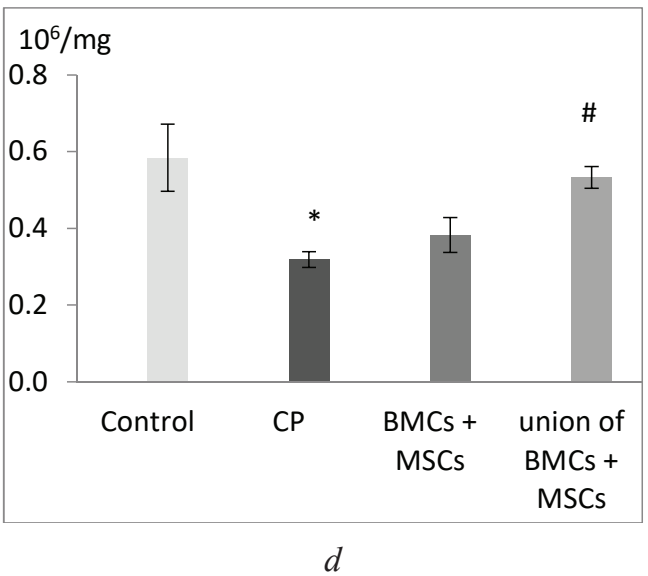

Fig. 1. Cellularity of bone marrow, lymphoid organs and proliferative activity of the murine cells: $a$-bone marrow cellularity; $b$ - cellularity of the thymus; $c$-- cellularity of the spleen; $d$ - mesenteric lymph nodes cellularity; control - control animals; $\mathrm{CP}$ - animals with cyclophosphamide treatment; BMCs $+\mathrm{MSCs}$ - animals treated with cyclophosphamide, bone marrow cells and thymic multipotent stromal cells separately; union of BMCs+MSCs - animals, treated with cyclophosphamide, union of bone marrow cells and thymic multipotent stromal cells

Notes: $*-p<0.05$ - the difference is significant compared to the group of normal mice

that received saline; \#-p<0.05 - compared to the group of mice with cyclophosphamide treatment; $+-p<0.05$ - compared to the group of mice with cyclophosphamide treatment that received bone marrow cells and thymic multipotent stromal cells separately

Under the impact of CP, the thymus weight was significantly decreased by $69.3 \%(p<0.005)$, the total number of thymocytes by $87.2 \%(p<0.005)$ and cellularity of the thymus by $63.6 \%$ $(p<0.005)$, indicating thymic involution. The application of the union of BMCs and MSCs did not affect these parameters. However, the co-transplantation BMCs and MSCs separately demonstrated the normalization of all thymic parameters (Fig. 1, b).

The weight and the relative mass of the spleen in mice, treated with $\mathrm{CP}$, were significantly increased after 12 days. At the same time, the number of splenocytes did not change and cellularity of the spleen even reduced. The characteristic change of these indexes allows to assume that the spleen enlargement under the impact of CP is the result of vascular disorders and edema of the lymphoid tissue. After co-transplantation spleen cellularity normalized significantly (Fig. 1, c). Thus, these experimental conditions reveal the positive effect of both type of cells on the spleen. 
When administering $\mathrm{CP}$, the cellularity of the mesenteric lymph nodes also decreased significantly by $44.8 \%(p<0.005)$ compared to the control group of intact mice. Both type of co-transplantation caused complete restoration of mesenteric lymph nodes cellularity (Fig. 1, d).

The level of spontaneous apoptosis of lymph node cells under the impact of the union of cells increased significantly compared with the control group of intact mice (Fig. 2, a). Data on the positive effects of separate co-transplantation on lymph node cells are confirmed by the results of the study of the cell cycle phases. The number of cells in the G0/G1 phases has significantly reduced, and in the $\mathrm{S}+\mathrm{G} 2 / \mathrm{M}$ phases has significantly increased (Fig. 2, b). While the union of BMCs and MSCs did not have such activity, it indicates the divergence in the effects of the studied cells. There are studies, in which a significant increase in the number of cells in the G2/M phase was observed in the non-adherent cells when MSCs and HSCs are co-cultured [18]. But in our experiment adherent cells were used.

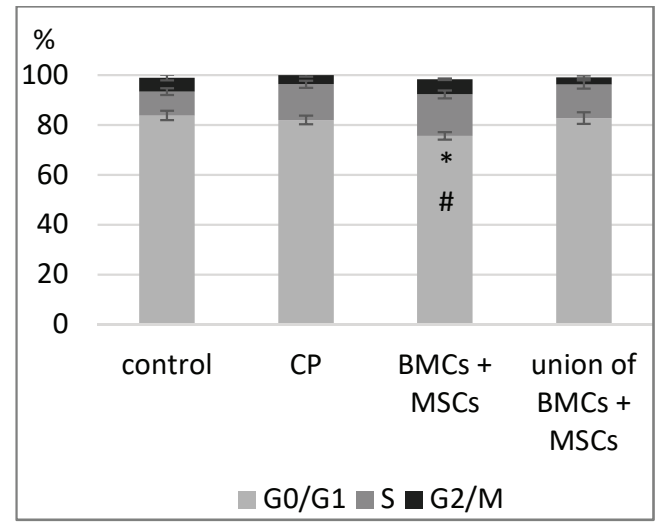

$a$

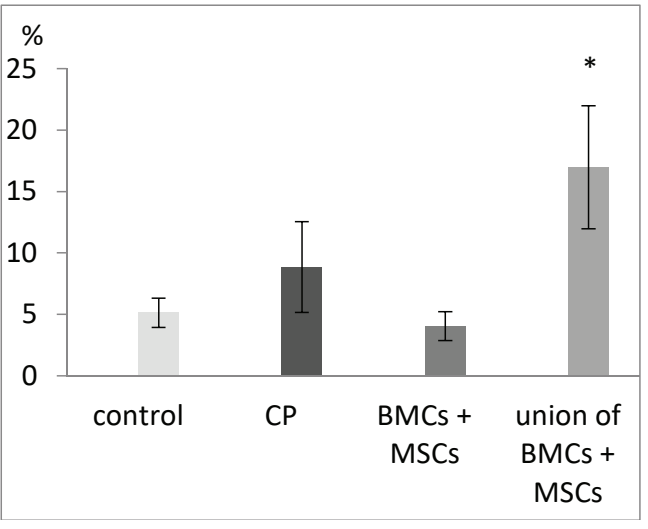

$b$

Fig. 2. Proliferative activity of the murine cells of mesenteric lymph nodes: $a$ - relative number of apoptotic cells; $b$ - relative number of cells G0/G1, S, G2/M cell cycle phase; control - control animals; $\mathrm{CP}$ - animals with cyclophosphamide treatment; $\mathrm{BMCs}+\mathrm{MSCs}$ - animals, treated with cyclophosphamide, bone marrow cells and thymic multipotent stromal cells separatly; union of BMCs+MSCs - animals, treated with cyclophosphamide, union of bone marrow cells and thymic multipotent stromal cells

Notes: ${ }^{*}-p<0.05$ - the difference is significant compared to the group of normal mice that received saline

Thus, as a result of cells administration into mice, treated with CP, showed partial normalization of cellular parameters in various organs of the immune system. The efficacy of MSCs as an inductors of HSCs at bone marrow transplantation may be explained by the contact interaction of HSCs and stromal cells, as well as by the production of cytokines and growth factors necessary for homing and differentiation of HSCs [19, 20].

The treatment with cyclophosphamide significantly decreased a hematocrit and hemoglobin level. BMCs and MSCs co-transplantation separately normalized the hematocrit and hemoglobin level in the blood (Fig. 3). Transplanted the union of BMCs and MSCs did not restore hematological parameters.

BMCs showed a greater activity in recovery of the hematocrit and hemoglobin level, and, at the same time, significantly increased the number of reticulocytes in the blood, indicating an active process of erythropoiesis [21]. A similar effect was observed during separate co-transplantation, but not after transplantation of the cells union. Thus, the obtained data may indicate that a subpopulation of non-adherent cells is responsible for the recovery.

The functional activity of the immune system underwent pronounced changes. Although the phagocytic activity of macrophages did not change, the bactericidal activity of peritoneal mac- 
rophages in the NBT test under the CP treatment was substantially increased. Under the impact of the transplanted union of BMCs and MSCs there was the normalization of bactericidal activity (Fig. 3, a), which did not happen during the separate co-transplantation of these cells. At the separate co-transplantation, there was a pronounced stimulating effect on the natural cytotoxicity of splenocytes (Fig. 3, b) and the delayed type hypersensitivity reaction (DTHR) (Fig. 3, $\boldsymbol{d}$ ) compared to the group of mice, receiving only $\mathrm{CP}$, a significant increase. The separate co-transplantation resulted in a pronounced suppressive effect on the blast transformation, induced by phytohemagglutinin (Fig. 3, ). After the application of the union of MSCs and BMCs, there were not any significant differences from the norm. The transplantation of the union of MSCs and BMCs had a suppressive effect on the number of antibody-producing cells in the spleen (Fig. 3, e).

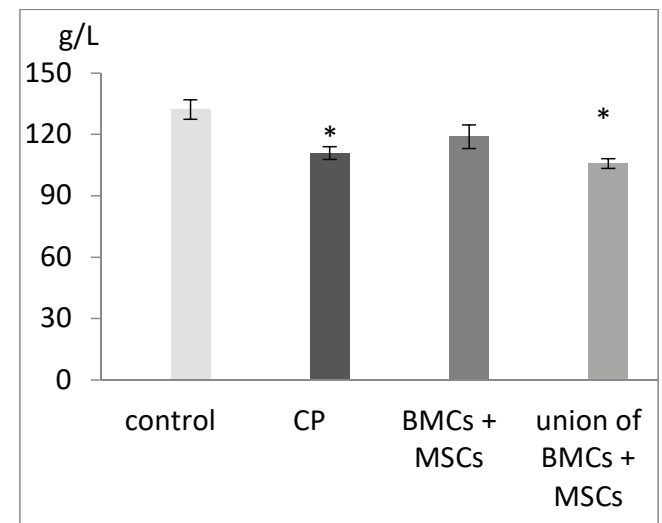

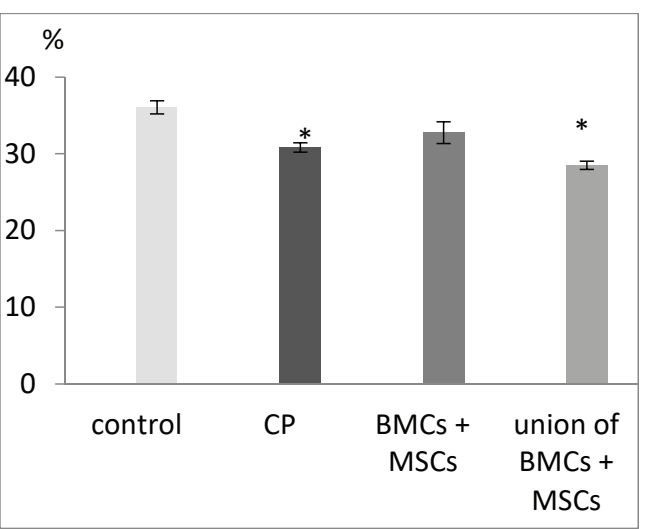

$b$

Fig. 3. Hematological parameters of mice: $a$ - hemoglobin level; $b$ - hematocrit; control - control animals; CP - animals with cyclophosphamide treatment; BMCs+MSCs - animals, treated with cyclophosphamide, bone marrow cells and thymic multipotent stromal cells separately; union of BMCs + MSCs - animals, treated with cyclophosphamide, union of bone marrow cells and thymic multipotent stromal cells

Notes: $*-p<0.05$ - the difference is significant compared to the group of normal mice that received saline

Separate co-transplantation of BMCs and MSCs is associated with the restoration of the number of bone marrow cells, thymus, spleen and lymph nodes with an increase in the proliferation index of lymph node cells by 1.4 times compared to control. It normalized the previous reduced concentration of hemoglobin and hematocrit in the blood, as well as BMCs. Co-transplantation had a suppressive effect on the blast transformation reaction, induced by phytohemagglutinin by 4.3 times, but showed a stimulating effect on DTHR response by 1.6 times compared to control.

After cells co-cultivation, the regenerative properties of cell grafts change, which is most likely due to the effect of cells contact interaction. Thus, co-transplantation of the union of BMCs and MSCs is associated with the restoration of the number of bone marrow cells, spleen and lymph nodes. But the level of spontaneous apoptosis of lymph node cells significantly increased by 3.3 times compared to control. It had no effect on hematological parameters, but is activated to impact the immune system. Thus, as a result of cells union administration showed normalization of the bactericidal activity of peritoneal macrophages, unlike the separate co-transplantation. This graft had a suppressive effect on the number of antibody-producing cells in the spleen by 4.2 times compared to control.

The findings of this study have to be seen in light of some limitations. The effect of co-transplantation of BMCs and MSCs on the recovery of the immune system cannot be assessed due to the biological characteristics of immunodeficiencies. Under the conditions of the experiment, we induced chemotherapeutic immunodeficiency by CP in mice. Therefore, for each immunopathology it is necessary to carry out individual selection of doses and combinations of cells for transplantation. 

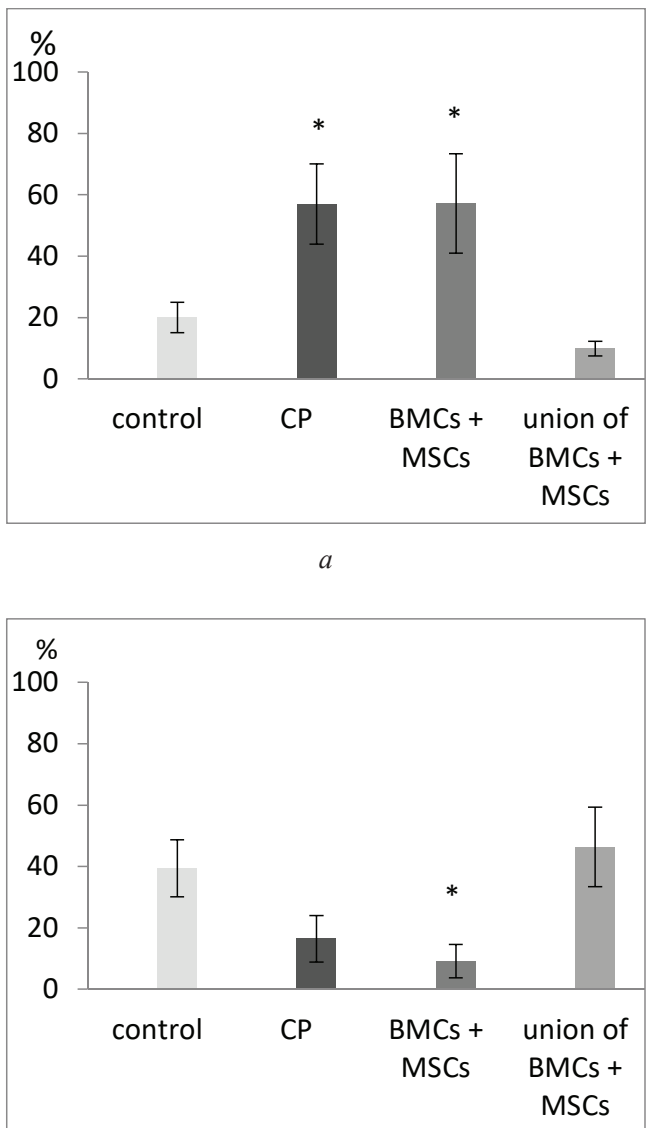

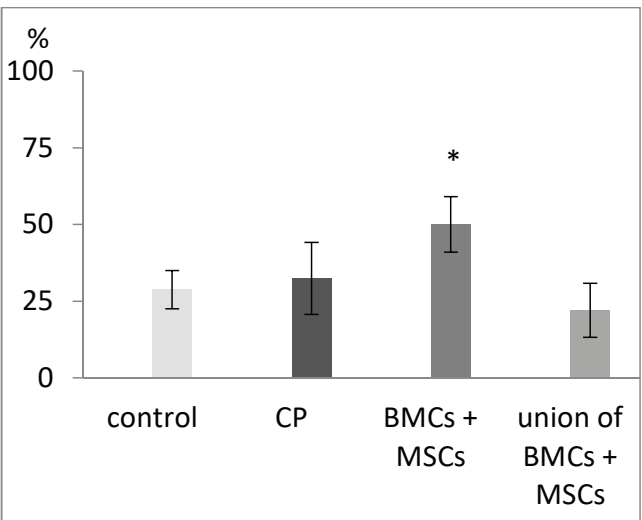

$b$

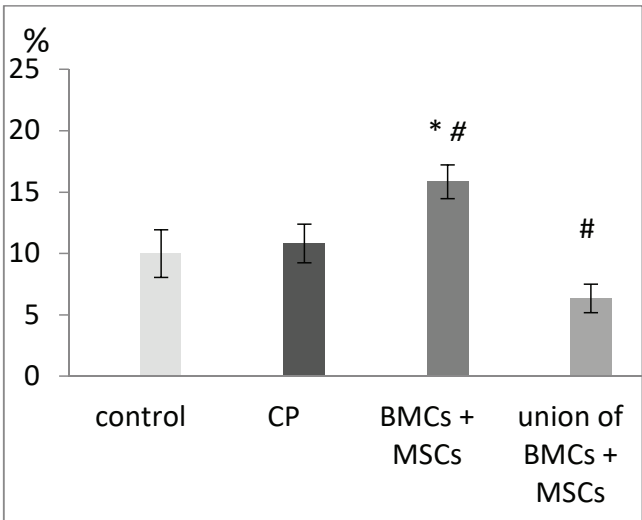

$d$

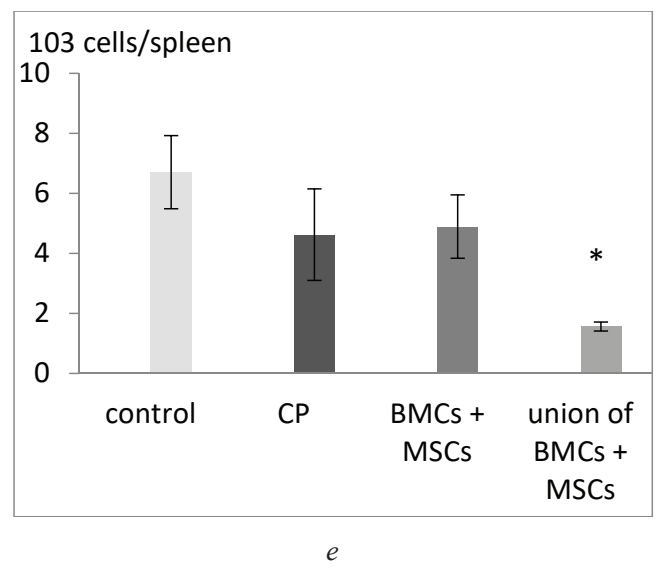

Fig. 4. Parameters of natural and adaptive immunity of mice:

a - bactericidal activity of peritoneal macrophages; $b$ - cytotoxic activity of splenocytes; $c$ - splenocytes blast transformation, induced by phytohemagglutinin; $d$ - the delayed type hypersensitivity;

$e$ - the number of antibody-producing cells in the spleen; control - control animals;

$\mathrm{CP}$ - animals with cyclophosphamide treatment; BMCs+MSCs - animals treated with cyclophosphamide, bone marrow cells and thymic multipotent stromal cells separately; union of BMCs+MSCs - animals, treated with cyclophosphamide, union of bone marrow cells and thymic multipotent stromal cells

Notes: $*-p<0.05$ - the difference is significant compared to the group of normal mice that received saline; \# - $p<0.05$ - compared to the group of mice with cyclophosphamide treatment 
The obtained data on the effectiveness of cellular grafts experimentally substantiate the possibility of their implementation in various immunopathologies and may be useful in elaborating new approaches to transplantation of HSCs and MSCs for clinical practice.

\section{Conclusions}

The effect of cell grafts of BMCs and thymic MSCs is method-dependent. Twelve days after the CP treatment, separate co-transplantation of BMCs and MSCs restored the number of bone marrow cells, thymus, spleen and lymph nodes. The concentration of hemoglobin and hematocrit in the blood was normalized. Transplantation suppressed the blast transformation reaction, induced by phytohemagglutinin by 4.3 times. DTHR was stimulated by 1.6 times compared to control.

The union of BMCs and MSCs administration into mice treated with CP showed normalization of cellular parameters in the bone marrow, spleen, lymph nodes. Unlike the separate transplantation, the bactericidal activity of peritoneal macrophages was normalized. The union of cells suppressed antibody-production in the spleen by 4.2 times compared to control. This method of co-transplantation stimulated apoptosis of lymph node cells by 3.3 times compared to control.

\section{Conflicts of interest}

The authors declare no conflicts of interest.

\section{References}

[1] Mucientes, A., Herranz, E., Moro, E., González-Corchón, A., Peña-Soria, M. J., Abasolo, L. et. al. (2021). Influence of Mesenchymal Stem Cell Sources on Their Regenerative Capacities on Different Surfaces. Cells, 10 (2), 481. doi: https://doi.org/ 10.3390/cells10020481

[2] Wu, X., Jiang, J., Gu, Z., Zhang, J., Chen, Y., Liu, X. (2020). Mesenchymal stromal cell therapies: immunomodulatory properties and clinical progress. Stem Cell Research \& Therapy, 11 (1). doi: https://doi.org/10.1186/s13287-020-01855-9

[3] Ho, Y.-H., Méndez-Ferrer, S. (2020). Microenvironmental contributions to hematopoietic stem cell aging. Haematologica, 105 (1), 38-46. doi: https://doi.org/10.3324/haematol.2018.211334

[4] Takam Kamga, P., Bazzoni, R., Dal Collo, G., Cassaro, A., Tanasi, I., Russignan, A. et. al. (2021). The Role of Notch and Wnt Signaling in MSC Communication in Normal and Leukemic Bone Marrow Niche. Frontiers in Cell and Developmental Biology, 8. doi: https://doi.org/10.3389/fcell.2020.599276

[5] Campinoti, S., Gjinovci, A., Ragazzini, R., Zanieri, L., Ariza-McNaughton, L., Catucci, M. et. al. (2020). Reconstitution of a functional human thymus by postnatal stromal progenitor cells and natural whole-organ scaffolds. Nature Communications, 11 (1). doi: https://doi.org/10.1038/s41467-020-20082-7

[6] Kreins, A. Y., Maio, S., Dhalla, F. (2020). Inborn errors of thymic stromal cell development and function. Seminars in Immunopathology, 43 (1), 85-100. doi: https://doi.org/10.1007/s00281-020-00826-9

[7] Kawashima, A., Yasuhara, R., Akino, R., Mishima, K., Nasu, M., Sekizawa, A. (2020). Engraftment potential of maternal adipose-derived stem cells for fetal transplantation. Heliyon, 6 (3), e03409. doi: https://doi.org/10.1016/j.heliyon.2020.e03409

[8] Lee, W., Wang, L., Yen, M., Hsu, P., Lee, Y., Liu, K. et. al. (2021). Resident vs nonresident multipotent mesenchymal stromal cell interactions with B lymphocytes result in disparate outcomes. STEM CELLS Translational Medicine, 10 (5), 711-724. doi: https:// doi.org/10.1002/sctm.20-0289

[9] Prockop, D. J., Bunnell, B. A., Phinney, D. G. (Eds.) (2008). Mesenchymal Stem Cells. Humana Press, 192. doi: https://doi.org/ 10.1007/978-1-60327-169-1

[10] Gregory, C. A., Grady Gunn, W., Peister, A., Prockop, D. J. (2004). An Alizarin red-based assay of mineralization by adherent cells in culture: comparison with cetylpyridinium chloride extraction. Analytical Biochemistry, 329 (1), 77-84. doi: https:// doi.org/10.1016/j.ab.2004.02.002

[11] Kim, W.-K., Jung, H., Kim, D.-H., Kim, E.-Y., Chung, J.-W., Cho, Y.-S. et. al. (2009). Regulation of adipogenic differentiation by LAR tyrosine phosphatase in human mesenchymal stem cells and 3T3-L1 preadipocytes. Journal of Cell Science, 122 (22), 4160-4167. doi: https://doi.org/10.1242/jcs.053009

[12] Nikolskiy, I., Nikolskaya, V., Demchenko, D., Zubov, D. (2016). Potentiation of directed osteogenic differentiation of thymic multipotent stromal cells by prior co-cultivation with thymocytes. Cell and Organ Transplantology, 4 (2), 220-223. doi: https:// doi.org/10.22494/cot.v4i2.59 
[13] Hassan, M., Said, R., Abdel-Rehim, M., Sadeghi, B., Al-Hashemi, S., Hassan, Z. (2008). Cyclophosphamide Pharmacokinetics in Mice: A Comparison Between Retro Orbital Sampling Versus Serial Tail Vein Bleeding. The Open Pharmacology Journal, 1 (1), 30-35. doi: https://doi.org/10.2174/1874143600701010030

[14] Haitov, R. M., Pinegin, B. V., Yarilin, A. A. (2009). Rukovodstvo po klinicheskoy immunologii. Diagnostika zabolevaniy immunnoy sistemy. Moscow: GEOTAR-Media, 352.

[15] Mosmann, T. (1983). Rapid colorimetric assay for cellular growth and survival: Application to proliferation and cytotoxicity assays. Journal of Immunological Methods, 65 (1-2), 55-63. doi: https://doi.org/10.1016/0022-1759(83)90303-4

[16] Sugiura, H., Sugiura, H., Nishida, H., Inaba, R., Mirbod, S. M., Iwata, H. (2001). Effects of different durations of exercise on macrophage functions in mice. Journal of Applied Physiology, 90 (3), 789-794. doi: https://doi.org/10.1152/jappl.2001.90.3.789

[17] Zhang, X., Goncalves, R., Mosser, D. M. (2008). The Isolation and Characterization of Murine Macrophages. Current Protocols in Immunology, 83 (1). doi: https://doi.org/10.1002/0471142735.im1401s83

[18] Jing, D., Fonseca, A. V., Alakel, N., Fierro, F. A., Muller, K., Bornhauser, M. et. al. (2010). Hematopoietic stem cells in co-culture with mesenchymal stromal cells - modeling the niche compartments in vitro. Haematologica, 95 (4), 542-550. doi: https:// doi.org/10.3324/haematol.2009.010736

[19] Rota, C., Morigi, M., Cerullo, D., Introna, M., Colpani, O., Corna, D. et. al. (2018). Therapeutic potential of stromal cells of non-renal or renal origin in experimental chronic kidney disease. Stem Cell Research \& Therapy, 9 (1). doi: https://doi.org/ 10.1186/s13287-018-0960-8

[20] Patel, D. M., Shah, J., Srivastava, A. S. (2013). Therapeutic Potential of Mesenchymal Stem Cells in Regenerative Medicine. Stem Cells International, 2013, 1-15. doi: https://doi.org/10.1155/2013/496218

[21] Demchenko, D. (2018). The effect of transplantation of bone marrow cells induced by the contact with thymus-derived multipotent stromal cells on the immune system of mice, regenerating after cyclophosphamide treatment. Cell and Organ Transplantology, 6 (2). doi: https://doi.org/10.22494/cot.v6i2.89

Received date 30.07.2021

(C) The Author(s) 2021

Accepted date 31.08.2021

Published date 04.09.2021

This is an open access article under the Creative Commons CC BY license

How to cite: Demchenko, D., Nikolskiy, I., Nikolskaya, V., Pelykh, N. (2021). Comparison of the effects of co-transplantation of bone marrow hematopoietic stem cells and thymic multipotent stromal cells on the immune system of mice depending on methods. EUREKA: Life Sciences, 5, 3-11. doi: https://doi.org/10.21303/2504-5695.2021.001993 